\title{
Experimental Evaluation of a MANET Testbed in Indoor Stairs Scenarios
}

\author{
Masahiro Hiyama*, Makoto Ikeda ${ }^{\dagger}$, Leonard Barolli ${ }^{\ddagger}$, Elis Kulla*, Fatos Xhafa ${ }^{\S}$ and Arjan Durresi ${ }^{\uparrow}$ \\ * Graduate School of Engineering \\ Fukuoka Institute of Technology (FIT) \\ 3-30-1 Wajiro-Higashi, Higashi-Ku, Fukuoka 811-0295, Japan \\ E-mail: mgm09007@bene.fit.ac.jp, eliskulla@yahoo.com \\ $\dagger$ Center for Asian and Pacific Studies \\ Seikei University \\ 3-3-1 Kichijoji-Kitamachi, Musashino-Shi, Tokyo 180-8633, Japan \\ E-mail: makoto.ikd@acm.org \\ $\ddagger$ Department of Information and Communication Engineering \\ Fukuoka Institute of Technology (FIT) \\ 3-30-1 Wajiro-Higashi, Higashi-Ku, Fukuoka 811-0295, Japan \\ E-mail: barolli@fit.ac.jp \\ $\S_{\text {Department of Languages and Informatics Systems Technical }}$ \\ University of Catalonia \\ Jordi Girona 1-3, 08034 Barcelona, Spain \\ E-mail: fatos@1si.upc.edu \\ IDepartment of Computer and Information Science \\ Indiana University Purdue University Indianapolis (IUPUI) \\ 723 W. Michigan Street SL 280 Indianapolis, IN 46202, USA \\ Email: durresi@cs.iupui.edu
}

\begin{abstract}
In recent years, Mobile Ad hoc Networks (MANETs) are continuing to attract the attention for their potential use in several fields. Mobility and the absence of any fixed infrastructure make MANETs very attractive for mobility and rescue operations and time-critical applications. In this paper, we present the implementation and analysis of our implemented MANET testbed considering the Optimized Link State Routing (OLSR) protocol. We consider two models. One when all the nodes are static and another one when one node is moving. The mobile node moves toward the destination at a regular speed and when arrives at the corner of stairs is stops for about three seconds. In this work, we assess the performance of our MANET testbed in terms of throughput and packet loss. From our experiments, we found that the OLSR protocol has a good performance when nodes are in stationary state. However, when the node moves the throughput is decreased. We observed that the number of packet loss increases after 2-hops for static model and after 1-hop for moving model. But, when the node is moving, the packet loss for 2-hops to 4-hops is almost the same.
\end{abstract}

\section{INTRODUCTION}

A Mobile Ad hoc Network (MANET) is a collection of wireless mobile terminals that are able to dynamically form a temporary network without any aid from fixed infrastructure or centralized administration. In recent years, MANET are continuing to attract the attention for their potential use in several fields. Mobility and the absence of any fixed infrastructure make MANET very attractive for mobility and rescue operations and time-critical applications.

Most of the work for MANETS has been done in simulation, as in general, a simulator can give a quick and inexpensive understanding of protocols and algorithms. However, experimentation in the real world are very important to verify the simulation results and to revise the models implemented in the simulator. A typical example of this approach has revealed many aspects of IEEE 802.11, like the gray-zones effect [1], which usually are not taken into account in standard simulators, as the well-known $n s-2$ simulator.

So far, we can count a lot of simulation results on the performance of MANET, e.g. in terms of end-to-end throughput, delay and packet loss. However, in order to assess the simulation results, real-world experiments are needed and a lot of testbeds have been built to date [2]. The baseline criteria usually used in real-world experiments is guaranteeing the 
repeatability of tests, i.e. if the system does not change along the experiments. How to define a change in the system is not a trivial problem in MANET, especially if the nodes are mobile.

In this paper, we concentrate on the performance analysis of a small MANET testbed of six laptops acting as nodes of MANET. We use Optimized Link State Routing (OLSR) protocol, which is a pro-active routing protocol, and it has been gaining great attention within the scientific community. Furthermore, the olsrd [3] software we have used in our experiments is the most updated software we have encountered.

In our previous work [4]-[7], we carried out many experiments with our MANET testbed. We proved that while some of the OLSR's problems can be solved, for instance the routing loop, this protocol still have the self-interference problem. There is an intricate inter-dependence between MAC layer and routing layer, which can lead the experimenter to misunderstand the results of the experiments. For example, the horizon is not caused only by IEEE 802.11 Distributed Coordination Function (DCF), but also by the routing protocol. We carried out the experiments with different routing protocols such as OLSR and B.A.T.M.A.N. and found that throughput of TCP were improved by reducing Link Quality Window Size (LQWS), but there were packet loss because of experimental environment and traffic interference. For TCP data flow, we got better results when the LQWS value was 10. Moreover, we found that the node join and leave operations affect more the TCP throughput and RTT than UDP.

In this work, different from our previous work, we investigate the performance of MANET testbed in indoor stairs scenarios. We implemented six MANET models and evaluated its performance considering throughput and packet loss parameters.

The structure of the paper is as follows. In Section II, we discuss the related work. In Section III, we introduce the MANET testbed design and implementation. In Section IV, we present experimental evaluation. Finally, conclusions are given in Section $\mathrm{V}$.

\section{RELATED WORK}

In [8], the authors analyse the performance of an outdoor ad-hoc network, but their study is limited to reactive protocols such as Ad hoc On Demand Distance Vector (AODV) and Dynamic Source Routing (DSR). The authors of [9], performs outdoor experiments of non standard pro-active protocols. Other ad-hoc experiments are limited to identify MAC problems, by providing insights on the one-hop MAC dynamics as shown in [10].

The closest work to ours is that in [11]. However, the authors did not care about the routing protocol. In [12], the disadvantage of using hysteresis routing metric is presented through simulation and indoor measurements. Our experiments are concerned with the interaction of transport protocols and routing protocol, for instance OLSR. Furthermore, we compare the performance of the testbed for six scenarios.

In [13], the authors presents an experimental comparison of OLSR using the standard hysteresis routing metric and the
Expected Transmission Count (ETX) metric in a 7 by 7 grid of closely spaced $\mathrm{Wi}-\mathrm{Fi}$ nodes to obtain more realistic results. The throughput results are similar to our previous work and are effected by hop distance [5].

\section{MANET TESTBED DESIGN AND IMPLEMENTATION}

\section{A. Target Environment}

We have implemented a MANET testbed which provides a realistic platform for analysing various aspect of these networks, including the different topology models. For our testbed, we make the following considerations.

- We consider an indoor environment in the stairs of our department building.

- We investigate the effect of mobility in the throughput and packet loss of MANET testbed.

- We constructed two experimental models: Model 1 (all nodes are in stationary state); Model 2 (only one intermediate node is moving). In Table I, we show the types of nodes for each experimental model.

- The mobile nodes move toward the destination at a regular speed. When the mobile node arrives at the corner point of the stairs, it stops for about three seconds.

- Experimental time is 10 seconds.

\section{B. OLSR}

The link state routing protocol that is most popular today in the open source world is OLSR from olsr.org. OLSR with Link Quality (LQ) extension and fisheye-algorithm works quite well. The OLSR protocol is a pro-active routing protocol, which builds up a route for data transmission by maintaining a routing table inside every node of the network. The routing table is computed upon the knowledge of topology information, which is exchanged by means of Topology Control (TC) packets. The TC packets in turn are built after every node has filled its neighbors list. This list contains the identity of neighbor nodes. A node is considered a neighbor if and only if it can be reached via a bi-directional link.

OLSR makes use of HELLO messages to find its one hop neighbors and its two hop neighbors through their responses. The sender can then select its Multi Point Relays (MPR) based on the one hop node which offer the best routes to the two hop nodes. By this way, the amount of control traffic can be reduced. Each node has also an MPR selector set which enumerates nodes that have selected it as an MPR node. OLSR uses TC messages along with MPR forwarding to disseminate neighbor information throughout the network. OLSR checks the symmetry of neighbor nodes by means of a 4-way handshake based on HELLO messages. This handshake is inherently used to compute the packet loss probability over a certain link. This can sound odd, because packet loss is generally computed at higher layer than routing one. However, an estimate of the packet loss is needed by OLSR in order to assign a weight or a state to every link. Host Network Address (HNA) messages are used by OLSR to disseminate network route advertisements in the same way that TC messages advertise host routes. 
OLSRv2 is currently being developed at IETF. It maintains many of the key features of the original protocol including MPR selection and dissemination. Key differences are the flexibility and modular design using shared components such as packet format packetbb and neighborhood discovery protocol.

In our OLSR code, a simple RFC-compliant heuristic is used [14] to compute the MPR nodes. Every node computes the path towards a destination by means of a simple shortestpath algorithm, with hop-count as target metric. In this way, a shortest path can result to be also not good, from the point of view of the packet error rate. Accordingly, recently olsrd has been equipped with the LQ extension, which is a shortestpath algorithm with the average of the packet error rate as metric. This metric is commonly called as the ETX, which is defined as $\operatorname{ETX}(i)=1 /(N I(i) \times L Q I(i))$. Given a sampling window $W, \mathrm{NI}(i)$ is the packet arrival rate seen by a node on the $i$-th link during $W$. Similarly, $\mathrm{LQI}(i)$ is the estimation of the packet arrival rate seen by the neighbor node which uses the $i$-th link. When the link has a low packet error rate, the ETX metric is higher. The LQ extension greatly enhances the packet delivery ratio with respect to the hysteresis-based technique [15].

\section{Testbed Description}

Our testbed is composed of six laptops machines. We constructed two experimental models. The experimental parameters are shown in Table II. In Fig. 1, all nodes are in a stationary state. We call this model STAIRS STA. In Fig. 2, only one node (node id \#6) is moving. The one-way trip time is about 80 seconds. We call this model STAIRS MOVE. The nodes position and movement are shown in Figs. 3(a), 3(b), 3(c), 3(d) and 3(e).

The operating system mounted on these machines is Ubuntu Linux with kernel 2.6.28 over, suitably modified in order to support the wireless cards. The wireless network cards are from Linksys. They are usb-based cards with and external antenna of $2 \mathrm{dBi}$ gain, transmitted power of $16+/-1 \mathrm{dBm}$ and receive sensitivity of $-80 \mathrm{dBm}$. We verified that the external antenna improves the quality of the first hop link, which is the link connecting the ad-hoc network. The driver can be downloaded from the web site in references [16], [17] ${ }^{1}$.

In our testbed, we have two systematic background or interference traffic we could not eliminate: the control traffic and the other wireless APs interspersed within the campus. The control traffic is due to the ssh program, which is used to remotely start and control the measurement software on the source node. The other traffic is a kind of interference, which is typical in an academic scenario.

\section{Testbed Interface}

Until now, all the parameters settings and editing were done by using command lines of bash shell (terminal), which resulted in many misprints and the experiments were repeated many times. In order to make the experiments easier, we

\footnotetext{
${ }^{1}$ As far as we know the latest kernel include rt2500usb driver. However, this driver does not work for ad-hoc mode.
}

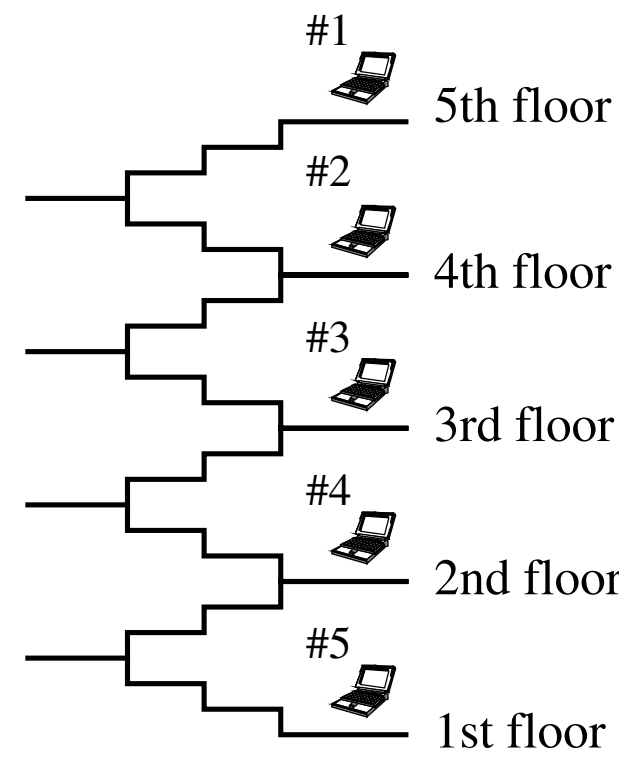

Fig. 1. Stairs stationary model (STAIRS STA).

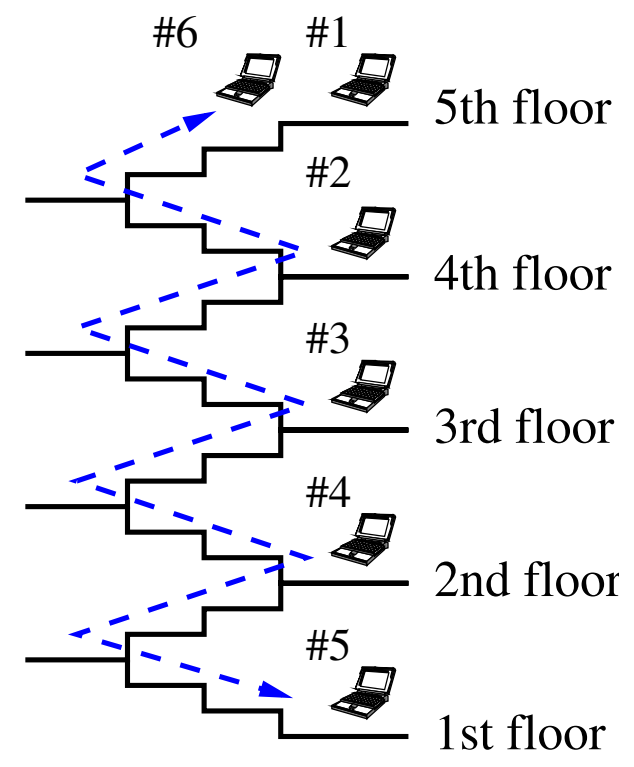

Fig. 2. Stairs move model (STAIRS MOVE, one node move).

TABLE I

TYPES OF NODES FOR EACH EXPERIMENTAL MODEL.

\begin{tabular}{l|c|c|c}
\hline \hline \multirow{2}{*}{ Model } & \multicolumn{2}{|c|}{ Number of moving nodes } & Num. of stati- \\
\cline { 2 - 3 } & Source node & Relay node & onary nodes \\
\hline \hline STAIRS STA & 0 & 0 & 5 \\
STAIRS MOVE & 0 & 1 & 5 \\
\hline
\end{tabular}

implemented a testbed interface. For the Graphical User Interface (GUI) we used wxWidgets tool and each operation is implemented by Perl language. wxWidgets is a cross-platform GUI and tools library for GTK, MS Windows and Mac OS.

We implemented many parameters in the interface such as 


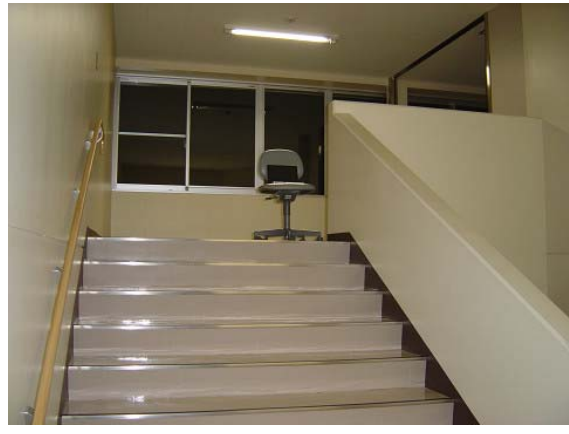

(a) Node ID \#1 on 5th floor

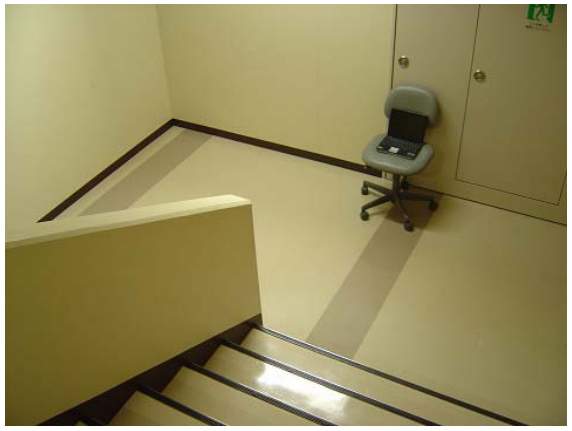

(b) Node ID \#2 on 4th floor

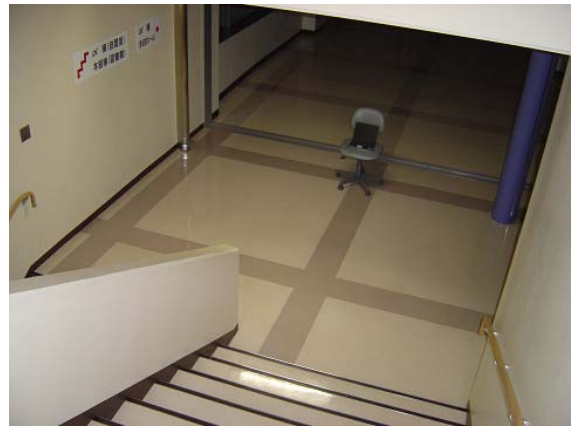

(c) Node ID \#3 on 3rd floor

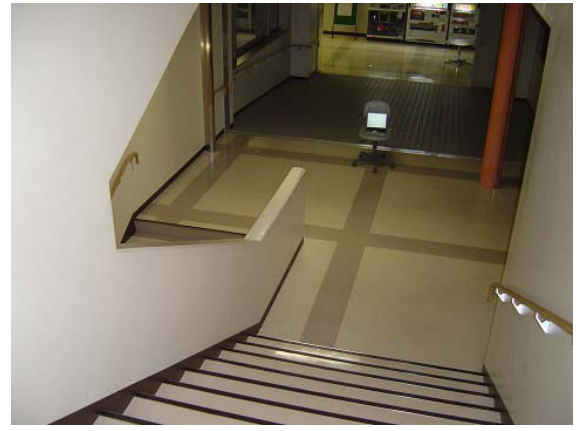

(d) Node ID \#4 on 2nd floor

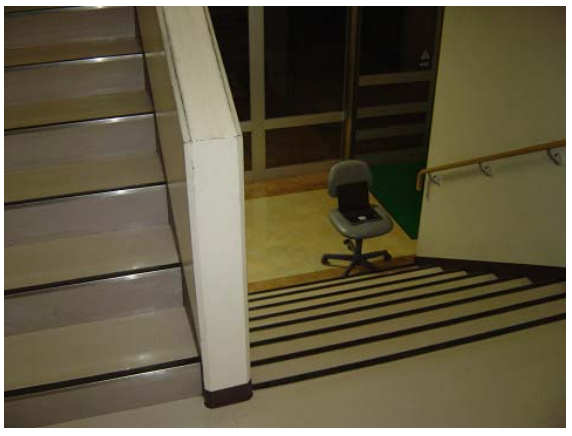

(e) Node ID \#5 on 1st floor

Fig. 3. Snapshot of each node.

TABLE II

EXPERIMENTAL PARAMETERS

\begin{tabular}{c||c}
\hline \multicolumn{1}{|c||}{ Function } & Value \\
\hline \hline Number of Nodes & 5 or 6 \\
MAC & IEEE 802.11 \\
Flow Type & CBR \\
Packet Rate & $122 \mathrm{pps}$ \\
Packet Size & $512 \mathrm{bytes}$ \\
Number of Trials & 30 \\
Duration & $10 \mathrm{sec}$ \\
Routing Protocol & OLSR \\
Link Quality Window Size & 10 \\
\hline
\end{tabular}

transmission duration, number of trials, source address, destination address, packet rate, packet size, LQWS, and topology setting function. We can save the data for these parameters in a text file and can manage in a better way the experimental conditions. Moreover, we implemented collection function of experimental data in order to make easier the experimenter's work.

\section{EXPERIMENTAL RESULTS}

\section{A. Experimental Settings}

The experimental parameters are shown in Table II. We study the impact of best-effort traffic for Mesh Topology (MT). In the MT scheme, the MAC filtering routines are not enabled. We collected data for two metrics: the throughput and packet loss. These data are collected by using the Distributed Internet Traffic Generator (D-ITG) [18], which is an opensource Internet traffic generator. D-ITG computes the packet loss as the number of lost packet divided by the effective number of sent packets.

The transmission rate of the data flows is $122 \mathrm{pps}=499.712$ Kbps, i.e. the packet size of the payload is 512 bytes. All experiments have been performed in indoor environment, in the stairs of our department building. All laptops are in radio range of each other. The experimental time for one experiment was about 10 seconds.

We measured the throughput of UDP, which is computed at the receiver. We estimated the packet loss to compute the link quality metrics, e.g. LQ. For OLSR, $w T_{\text {HELLO }}<T_{\operatorname{Exp}}$, where $T_{\operatorname{Exp}}$ is the total duration of the experiment, i.e., in our case, $T_{\operatorname{Exp}}=300$ seconds, and $T_{\text {HELLO }}$ is the rate of the HELLO messages. However, the testbed was turned on even in the absence of measurement traffic. Therefore, the effective $T_{\text {Exp }}$ was much greater.

As MAC protocol, we used IEEE 802.11b. The transmission power was set in order to guarantee a coverage radius equal to the maximum allowed geographical distance in the network. Since we were interested mainly in the performance of the routing protocol, we kept unchanged all MAC parameters, such as the carrier sense, the retransmission counter, the contention window and the RTS/CTS threshold. Moreover, the channel central frequency was set to $2.412 \mathrm{GHz}$ (channel 1). In regard to the interference, it is worth noting that, during our tests, almost all the IEEE 802.11 spectrum had been used by other access points disseminated within the campus. In general, the interference from other access points is a noncontrollable parameter. 
TABLE III

MEDIAN THROUGHPUT (KBPS).

\begin{tabular}{c|c|c|c|c|c}
\hline \hline \multirow{2}{*}{ Model } & \multicolumn{5}{|c}{ Source and destination node id } \\
\cline { 2 - 6 } & $1 \rightarrow 2$ & $1 \rightarrow 3$ & $1 \rightarrow 4$ & $1 \rightarrow 5$ & $1 \rightarrow 6$ \\
\hline \hline STAIRS STA, CBR & 499.7120 & 499.7120 & 450.3040 & 308.9237 & - \\
STAIRS MOVE, CBR & 499.3024 & 172.1458 & 182.5451 & 110.1302 & 294.6095 \\
\hline
\end{tabular}

TABLE IV

MEdiAn NUMBER OF PACKET LOSS (PKT/SEC).

\begin{tabular}{c|c|c|c|c|c}
\hline \hline Model & \multicolumn{5}{|c}{ Source and destination node id } \\
\cline { 2 - 6 } & $1 \rightarrow 2$ & $1 \rightarrow 3$ & $1 \rightarrow 4$ & $1 \rightarrow 5$ & $1 \rightarrow 6$ \\
\hline \hline STAIRS STA, CBR & 0 & 0.6994 & 8.9843 & 8.3417 & - \\
STAIRS MOVE, CBR & 0.1121 & 12.7950 & 7.8824 & 12.1738 & 13.3493 \\
\hline
\end{tabular}

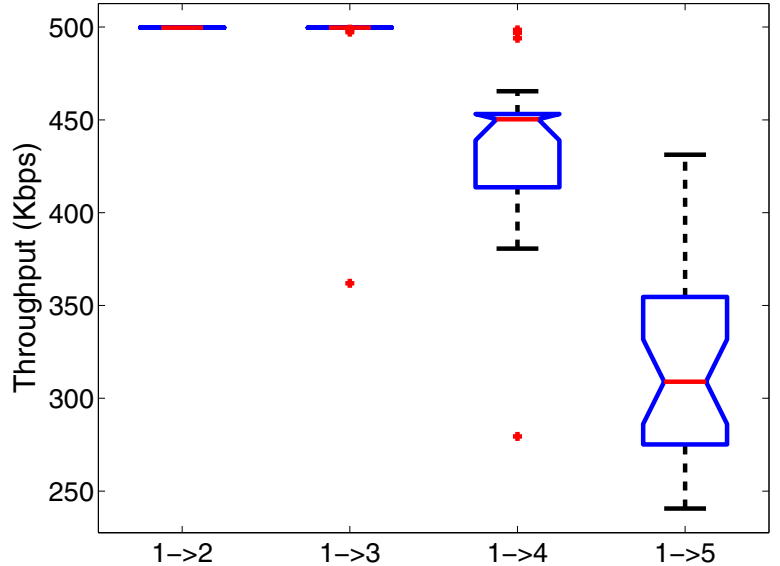

(a) STAIRS STA

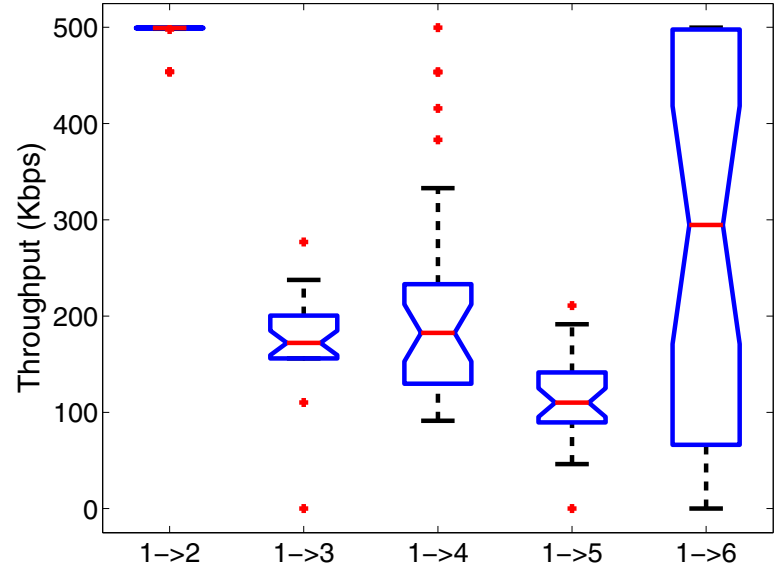

(b) STAIRS MOVE

Fig. 4. Throughput results.

\section{B. Experimental Measurements}

Here, we show the measured data by the box and whisker plot of the metrics according to the model types. Box and whisker plot is a convenient way to show groups of numerical data by lower quartile (Q1), median (Q2), upper quartile (Q3), and the outliers. In the plot, the bottom and top of the box are always 25th and 75th percentile (Q1 and Q3, respectively), and the band near the middle of the box is always the median (Q2). The end of the whiskers can represent the lowest datum which is still within 1.5 inter-quartile range of the lower quartile, and the highest datum which is still within 1.5 inter-quartile range of the upper quartile.

In Fig. 4(a) and Fig. 4(b), the horizontal axis show the hop distance $(1 \rightarrow 2$ means source node id $\rightarrow$ destination node $i d$ ) and the vertical axis shows the throughput (Kbps), which is computed at the receiver. In order to show the range of variability of the data, we also report the plot of the metrics according to the model types, as shown in Tables III and IV.

As shown in Fig. 4(a), there are few oscillations for STAIRS STA model for 2-hops and 3-hops. However, when as destination node is node \#5, the throughput is decreased about 40 percent compared with stable bitrate. In case of STAIRS MOVE model (Fig. 4(b)), the throughput is lower than STAIRS STA model (about less that $50 \%$ for more than 2-hops). From these results, we see that the OLSR protocol has a good performance for 1-hop and 2-hops (destination nodes are node \#2 and node \#3). However, the performance is not good when the destination node is in the 1st floor (4-hops).

Considering packet loss parameter, the number of packet loss increases after node $1 \rightarrow 3$ (2-hops) in Fig. 4(b) and after 1-hop in Fig. 5(b). It seems that the topology is very dynamic when node \#6 is moving. When node \#6 is moving, the packet loss for 2-hops to 4-hops is almost the same. We see that a direct symmetric link exists between nodes 1 and 3, and OLSR chooses correctly 2-hops route (1-6-3).

\section{CONCLUSIONS}

In this paper, we carried out experiments in indoor stairs environmet by using our implemented MANET testbed consisting of six nodes. We used OLSR protocol for experimental evaluation.

In our experiments, we considered two models: STAIRS STA and STAIRS MOVE. In STAIRS STA, all nodes are in a stationary state. In STAIRS MOVE, only one node is moving. We assessed the performance of our MANET testbed in terms of throughput and packet loss.

From our experiments, we found the following results. 


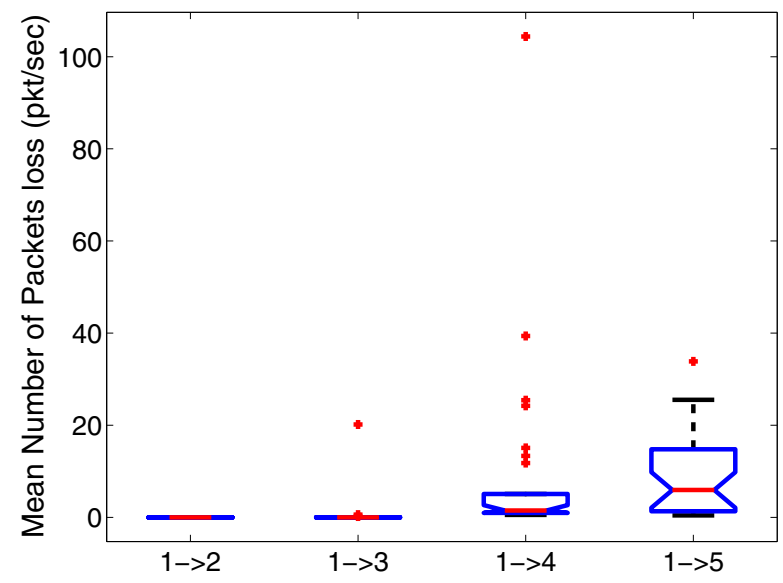

(a) STAIRS STA

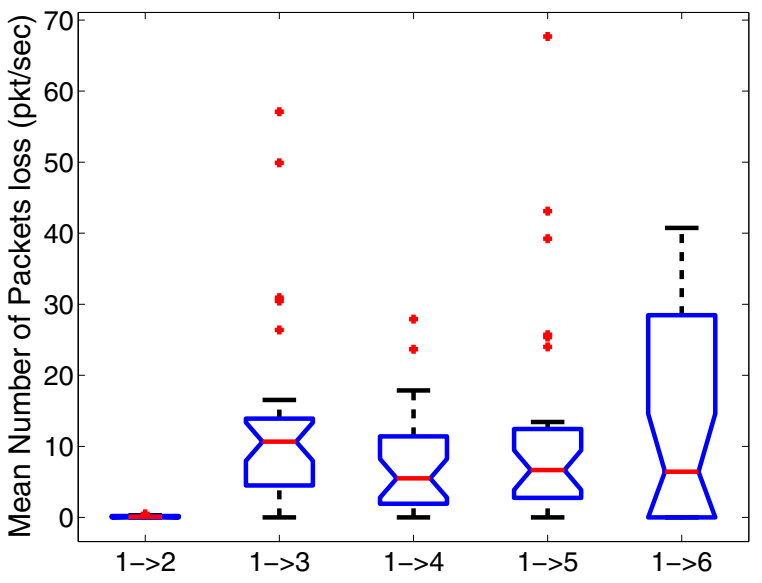

(b) STAIRS MOVE

Fig. 5. Mean number of packet loss results.

- There are few oscillations for STAIRS STA model for 2-hops and 3-hops. However, when as destination node is node \#5, the throughput is decreased about 40 percent compared with stable bitrate.

- In case of STAIRS MOVE model, the throughput is lower than STAIRS STA model (about less that $50 \%$ for more than 2-hops).

- The OLSR protocol has a good performance for 1-hop and 2-hops. However, the performance is not good for 4-hops.

- The number of packet loss increases after 2-hops for STAIRS STA Model and after 1-hop for STAIRS MOVE model. It seems that the topology is very dynamic when node \#6 is moving. When node \#6 is moving, the packet loss for 2-hops to 4-hops is almost the same.

These experiments where performed using a single flow through the network. In the future, we would like to consider the case of multiple flows. Moreover, we would like to compared the results with other routing protocols and extend our testbed.

\section{ACKNOWLEDGEMENT}

This work is supported by a Grant-in-Aid for scientific research of Japan Society for the Promotion of Science (JSPS). The authors would like to thank JSPS for the financial support.

\section{REFERENCES}

[1] H. Lundgren, E. Nordstrom, and C. Tschudin, "Coping with Communication Gray Zones in IEEE 802.11b based Ad Hoc Networks", Proc. of the 5-th ACM International Workshop on Wireless Mobile Multimedia (WOWMOM-2002), pp. 49-55, 2002.

[2] W. Kiess and M. Mauve, "A Survey on Real-world Implementations of Mobile Ad-hoc Networks", Ad Hoc Networks, Vol. 5, No. 3, pp. 324-339, 2007.

[3] A. Tonnesen, "OLSRd: Implementation Code of the OLSR", Available on line at http://www.olsr.org/.

[4] L. Barolli, M. Ikeda, G. De Marco, A. Durresi, and F. Xhafa, "Performance Analysis of OLSR and BATMAN Protocols Considering Link Quality Parameter", Proc. of IEEE AINA-2009, pp. 307-314, May 2009.
[5] G. De Marco, M. Ikeda, T. Yang, and L. Barolli, "Experimental Performance Evaluation of a Pro-active Ad-hoc Routing Protocol in Outdoor and Indoor Scenarios", Proc. of IEEE AINA-2007, pp. 7-14, May 2007.

[6] M. Ikeda, L. Barolli, G. De Marco, T. Yang, and A. Durresi, "Experimental and Simulation Evaluation of OLSR Protocol for Mobile Ad-hoc Networks", Proc. of NBiS-2008, pp. 111-121, September 2008.

[7] M. Hiyama, M. Ikeda, L. Barolli, G. De Marco, F. Xhafa, and A. Durresi, "Mobility Effects in Mobile Ad Hoc Networks", Proc. of International Workshop on Network Traffic Control, Analysis and Applications (NTCAA-2009), Vol. 2, pp. 679-684, December 2009.

[8] D. A. Maltz, J. Broch, and D. B. Johnson, "Lessons from a Fullscale Multihop Wireless Ad Hoc Network Testbed", IEEE Personal Communications, Vol. 8, No. 1, pp. 8-15, February 2001.

[9] R. S. Gray, D. Kotz, C. Newport, N. Dubrovsky, A. Fiske, J. Liu, C. Masone, S. McGrath, and Y. Yuan, "Outdoor Experimental Comparison of Four Ad Hoc Routing Algorithms", Proc. MSWiM-2004, pp. 220-229, 2004.

[10] G. Anastasi, E. Borgia, M. Conti, and E. Gregori, "IEEE 802.11b Ad Hoc Networks: Performance Measurements", Cluster Computing, Vol. 8, No. 2-3, pp. 135-145, 2005.

[11] V. Kawadia and P. R. Kumar, "Experimental Investigations into TCP Performance over Wireless Multihop Networks", Proc. of E-WIND-2005, pp. 29-34, 2005.

[12] T. Clausen, G. Hansen, L. Christensen, and G. Behrmann, "The Optimized Link State Routing Protocol, Evaluation through Experiments and Simulation", Proc. of IEEE Symposium on Wireless Personal Mobile Communications, Available on line at http://hipercom.inria.fr/olsr/wpmc01.ps, September 2001.

[13] D. Johnson and G. Hancke, "Comparison of Two Routing Metrics in OLSR on a Grid based Mesh Network", Ad Hoc Networks, Vol. 7, No. 2, pp. 374-387, March 2009.

[14] T. Clausen and P. Jacquet, "Optimized Link State Routing Protocol (OLSR)", RFC 3626 (Experimental), 2003.

[15] D. S. J. D. Couto, D. Aguayo, J. Bicket, and R. Morris, "A High Throughput Path Metric for Multi-hop Wireless Routing", Proc. of MobiCom-2003, pp. 134-146, 2003.

[16] Rt2x00 Project, Available on line at http://rt2x00.serialmonkey.com/.

[17] Ralink RT2570 USB Enhanced Driver, Available on line at http://homepages.tu-darmstadt. de/ p p_larbig/wlan/.

[18] A. Botta, A. Dainotti, and A. Pescape, "Multi-protocol and Multiplatform Traffic Generation and Measurement", Proc. of INFOCOM-2007, Demo Session, May 2007. 